\title{
Fungos associados a grãos de café (Coffea arabica L.) beneficiados no sudoeste da Bahia*
}

\author{
Gabriel Fernandes Pinto Ferreira ${ }^{1}$, Quelmo Silva de Novaes ${ }^{1}$, Luis Roberto Batista ${ }^{2}$, Sandra Elizabeth de Souza ${ }^{1}$, \\ Gileno Brito de Azevedo ${ }^{1,}$ Daiani Maria da Silva ${ }^{3}$
}

\begin{abstract}
${ }^{1}$ Universidade Estadual do Sudoeste da Bahia, Departamento de Fitotecnia e Zootecnia, Cx. Postal 95, 45031-900, Vitória da Conquista, BA; ${ }^{2}$ Universidade Federal de Lavras, Departamento de Ciência dos Alimentos, Cx. Postal 3037, 37200-000, Lavras, MG. ${ }^{3}$ Universidade Federal de Lavras, Departamento de Biologia, Cx. Postal 3037, 37200-000, Lavras, MG; *Parte da Dissertação de Mestrado do primeiro autor, apresentada à Universidade Estadual do Sudoeste da Bahia em junho de 2010.

Autor para correspondência: Quelmo Silva de Novaes (qsnovaes@uesb.edu.br)

Data de chegada: 22/10/2010. Aceito para publicação em: 16/03/2011.
\end{abstract}

\section{RESUMO}

Ferreira, G.F.P.; Novaes, Q.S.; Batista, L.R.; Souza, S.E.; Azevedo, G.B. Fungos associados a grãos de café (Coffea arabica L.) beneficiados no sudoeste da Bahia. Summa Phytopathologica, v.37, n.3, p.98-102, 2011.

Diversos fatores podem interferir na qualidade do café, especialmente aqueles relacionados às etapas pós-colheita de processamento e secagem. Algumas espécies de fungos podem se associar a grãos de café durante a pós-colheita, podendo ocasionar alterações indesejáveis. O objetivo deste estudo foi avaliar a influência dos processamentos via seca (natural), seco em terreiro de terra, e via úmida (despolpado), seco em terreiro de cimento, tradicionalmente empregados na região sudoeste da Bahia, na incidência de fungos em grãos de café beneficiados produzidos na safra 2007/2008. O experimento consistiu de 4 tratamentos: a) café natural de Barra do Choça; b) café natural de Encruzilhada; c) café despolpado de Barra do Choça e d) café despolpado de
Encruzilhada; e 5 repetições. Foram coletadas 20 amostras de grãos de café oriundas de diferentes propriedades cafeeiras nestes municípios. Os resultados obtidos foram avaliados pelo teste de médias t de Bonferroni a $5 \%$ de probabilidade. Houve diferença estatística significativa entre os tratamentos analisados para a infestação fúngica. Os gêneros detectados foram: Aspergillus, Penicillium e Fusarium, sendo que o gênero Aspergillus foi o de maior incidência, no qual foram identificadas oito espécies: Aspergillus ochraceus, A. niger, A. flavus, A. foetidus, A. tubingensis, A. auricomus, A. sojae e A. oryzae. Foi detectada a maior incidência de fungos em grãos de café oriundos de processamento natural do que de processamento despolpado.

Palavras-chave adicionais: infestação fúngica, processamento, pós-colheita

\section{ABSTRACT}

Ferreira, G.F.P.; Novaes, Q.S.; Batista, L.R.; Souza, S.E.; Azevedo, G.B. Fungi associated with coffee beans (Coffea arabica L.) benefited in the southwest of Bahia, Brazil. Summa Phytopathologica, v.37, n.3, p.98-102, 2011.

Several factors can affect the quality of coffee, especially those related to the post-harvest stages of processing and drying. Some fungal species can associate with coffee beans during post-harvest, leading to undesirable alterations. The aim of this study was to evaluate the influence of dry (natural) processing, on-land drying, and humid (pulped) drying on cement floors, which are methods traditionally used in the southwestern region of Bahia State, Brazil, on the incidence of fungi on benefited coffee beans produced in the 2007/2008 harvest. The experiment consisted of 4 treatments: a) natural coffee of Barra do Choça; b) natural coffee of Encruzilhada; c) pulped coffee of
Barra do Choça and d) pulped coffee of Encruzilhada; and 5 replicates. Twenty samples of coffee beans were collected from different coffee growers in the above-mentioned cities. Results were evaluated using Bonferroni's $\mathrm{T}$ test at $5 \%$ probability. Significant differences were found between the analyzed treatments for fungal infestation. The genera of detected fungi were: Aspergillus, Penicillium and Fusarium, and Aspergillus had the highest incidence, with eight species identified: Aspergillus ochraceus, A. niger, A. flavus, A. foetidus, A. tubingensis, A. auricomus, A. sojae and A. oryzae. The incidence of fungi was higher in naturally processed than in pulped coffee beans.

Keywords: fungal infestation, processing, post-harvest

Os fungos filamentosos se encontram associados aos frutos e grãos de café (Coffea arabica L.) durante todo o ciclo produtivo e podem, sob condições específicas, causar perdas de qualidade produzindo odores e sabores desagradáveis e em alguns casos produzir micotoxinas, que comprometem a segurança do produto final (9). A incidência de fungos em café pode ser a principal causa para as diferenças de qualidade de bebida entre as regiões produtoras (1). As espécies de fungos encontradas em cafés brasileiros pertencem principalmente aos gêneros Aspergillus, Penicillium, Cladosporium e Fusarium $(18,20)$. Algumas espécies de Aspergillus e Penicillium estão associadas à produção de micotoxinas (11). A principal micotoxina estudada em café é a ocratoxina A (OTA) e sua presença foi relatada associada à presença do fungo Aspergillus ochraceus $(8,10,28)$.

Os fungos produtores de micotoxinas se encontram presentes nos 
ambientes das lavouras, preparo e armazenamento do café e sua relação com a qualidade e a segurança do produto final depende das condições ambientais, do manejo da cultura e do processamento pós-colheita (5). A infestação dos grãos de café pode ocorrer nas fases de processamento, principalmente durante a lavagem, fermentação e secagem $(28,30)$. A secagem é uma das rotas de infestação por espécies de Aspergillus ocratoxigênicos e consequentemente de contaminação pela ocratoxina A (7). O tipo de terreiro de secagem de café é importante nesta contaminação, sendo o terreiro de terra o mais favorável (19).

No Estado da Bahia, a cafeicultura apresenta importância considerável na economia agrícola, sendo a região sudoeste produtora de cafés de alta qualidade, com parte dessa produção direcionada à exportação para diversos países consumidores (13). Nesta região, os manejos pós-colheita do café, tradicionalmente empregados, são via seca (natural), na qual a secagem de grande parte da produção cafeeira é realizada em terreiros de terra, e a via úmida, representada principalmente pela prática do despolpamento com a secagem dos grãos em terreiro de cimento. O objetivo deste trabalho foi avaliar a composição fúngica de grãos de café processados por via seca (natural) e via úmida (despolpado) oriundos dos municípios de Barra do Choça e Encruzilhada localizados na região sudoeste da Bahia.

\section{MATERIAL E MÉTODOS}

Foram coletadas 10 amostras de cafés beneficiados (Coffea arabica L.) oriundos de processamento natural (via seca), com secagem em terreiro de terra, e 10 amostras de processamento despolpado (via úmida), com secagem em terreiro de cimento, perfazendo um total de 20 amostras. As coletas foram realizadas em diferentes propriedades cafeeiras, nos municípios de Barra do Choça e Encruzilhada, localizados na região sudoeste da Bahia. Cada amostra foi constituída de aproximadamente $1 \mathrm{~kg}$ de café beneficiado da safra 2007/2008.

A análise da composição fúngica foi realizada no Laboratório de Fitopatologia da Universidade Estadual do Sudoeste da Bahia UESB por Blotter test (29) adaptado para os conceitos de detecção e de isolamento de fungos em alimentos armazenados. Foram coletados ao acaso 200 grãos de café por amostra. Foi realizada desinfestação superficial em um lote de 100 grãos com hipoclorito de sódio a $1 \%$, para conhecer a composição fúngica interna, e outro lote de 100 grãos não foi desinfestado superficialmente, para permitir o conhecimento da composição fúngica externa. $\mathrm{O}$ plaqueamento dos grãos foi realizado em placas de Petri acrílicas, contendo duas folhas de papel filtro esterilizado e umedecidas com água destilada e esterilizada. A incubação das placas foi realizada em câmara BOD, por um período de sete dias, a uma temperatura de $23{ }^{\circ} \mathrm{C}$, com fotoperíodo de 12 horas, até a detecção de micélio e de estruturas reprodutivas dos fungos. As observações referentes aos grãos colonizados foram realizadas com auxílio de microscópio estereoscópico, avaliando-se a incidência e a severidade da infestação fúngica.

A Incidência (I) se refere à presença ou não de fungos nos grãos e foi expressa em porcentagem. A severidade da infestação fúngica se refere à área da superfície do grão infestada por colônia de fungo, sendo determinada por meio de escala proposta por Prabhu \& Bedendo (23) e adaptada para café por Freitas (15) na qual se atribuiu as seguintes notas: $0=$ sem infestação da superfície do grão evidente; 1 = colônias atingindo até $25 \%$ da superfície do grão; $2=$ de 26 a $50 \% ; 3=$ de 51 a $75 \%$ e $4=$ colônias atingindo mais de $75 \%$ da superfície do grão. Com os dados de severidade da infestação nos grãos e da Incidência (I) foi calculado o Índice de Severidade da Infestação (ISI) pela seguinte fórmula:

ISI $=\Sigma($ Nota $* \mathrm{I}) / \mathrm{n} *$ nota máxima, onde:

ISI = Índice de Severidade da Infestação;

I = Incidência de grãos infestados;

$\mathrm{n}=$ número de grãos analisados.

A identificação dos gêneros dos fungos conidiais foi realizada segundo a chave taxonômica descrita por Barnett \& Hunter (2) sendo observados os aspectos morfológicos e coloração das colônias nos grãos de café em microscópio estereoscópico, bem como características morfológicas dos conídios em microscópio óptico.

$\mathrm{O}$ isolamento dos fungos do gênero Aspergillus foi realizado em meio de cultura DG18 (Dichloran Glicerol 18\%) a partir daqueles que cresceram nos grãos plaqueados e incubados. Posteriormente procedeuse a purificação das culturas por repicagens sucessivas em meio de cultura MA (Agar Malte). A partir das culturas puras, as espécies do gênero Aspergillus foram incubadas em meio CYA (Czapeck Yeast Agar) a $25^{\circ} \mathrm{C}$ e $37{ }^{\circ} \mathrm{C}$ e MEA (Agar Extrato de Malte) a $25^{\circ} \mathrm{C}$ por 7 dias no Laboratório de Micologia e Micotoxinas da Universidade Federal de Lavras - UFLA, onde foi realizada a identificação das espécies através da observação das características macroscópicas e microscópicas de acordo com Klich (16).

O delineamento experimental foi inteiramente casualizado com quatro tratamentos: a) café natural de Barra do Choça; b) café natural de Encruzilhada; c) café despolpado de Barra do Choça e d) café despolpado de Encruzilhada, e com cinco repetições. Foram realizados dois experimentos independentes (grãos não desinfestados e grãos desinfestados), utilizado cada amostra como uma repetição. A análise estatística foi realizada para os fatores Incidência (I) e Índice de Severidade da Infestação (ISI), procedendose a análise de variância e a comparação das médias dos tratamentos pelo teste $\mathrm{t}$ de Bonferroni a $5 \%$ de probabilidade, utilizando-se o programa estatístico SISVAR 4.3 (14). Os dados de Índice de Severidade da Infestação (ISI) foram transformados em $\sqrt{x}+0,5$ para serem normalizados e homogeneizados, sendo que os valores de cada gênero e de algumas espécies encontradas foram considerados como uma variável.

\section{RESULTADOS E DISCUSSÃO}

Foram detectados fungos dos gêneros Aspergillus, Penicillium e Fusarium, em infestação e/ou infecção mista ou isoladamente, associados aos grãos de café, não-desinfestados e desinfestados, oriundos dos diferentes locais de origem e tipos de processamento pós-colheita. Dentro do gênero Aspergillus, foram identificadas as espécies A. ochraceus, A. niger, A. flavus, A. foetidus, A. tubingensis, A. auricomus, A. sojae e A. oryzae. A Incidência (I) e o Índice de Severidade da Infestação (ISI) das espécies Aspergillus ochraceus, A. niger e $A$. flavus, em grãos de café não-desinfestados e desinfestados, foram avaliados pelo fato destas serem potenciais produtoras de micotoxinas.

Conforme os resultados obtidos para I ISI de fungos em grãos de café não-desinfestados, observados nas Tabelas 1 e 2, respectivamente, verifica-se que houve diferença estatística significativa entre os tipos de processamento dos cafés oriundos dos municípios de Barra do Choça e Encruzilhada, para os gêneros Aspergillus e Penicillium, sendo maiores em processamento natural seco em terreiro de terra do que em processamento despolpado seco em terreiro de cimento. Esses 
resultados evidenciam que a contaminação fúngica é dependente do processamento pós-colheita adotado, sendo que a prática do despolpamento e secagem em terreiro de cimento sofreu uma menor contaminação fúngica que a prática do processamento natural com secagem em terreiro de terra.

O maior risco de exposição do café à contaminação por fungos toxigênicos e ocratoxina A foi caracterizado pelo contato do fruto com o solo, constituído pelo café de varrição e por manejo póscolheita inadequado durante a secagem em terreiro de terra (3). Assim, para a implementação de medidas de controle que possam prevenir a infestação fúngica e contaminação por ocratoxina A em frutos e grãos de café, deve-se evitar que estes entrem em contato direto com o solo, pois este representa uma importante fonte de contaminação (19). Trabalho realizado por Suárez-Quiroz et al. (27), demonstrou que $80 \%$ dos grãos analisados do processamento via úmida (despolpado), $72 \%$ do processamento mecânico (descascado) e $92 \%$ do processamento via seca (natural) estavam contaminados com fungos dos gêneros Aspergillus, Penicillium, Cladosporium, Fusarium, Mucor, Eurotium, Goetrichum e Botrytis.

A presença dos fungos na parte externa dos grãos de café indica uma contaminação do ambiente em que foram produzidos e processados (15). Chalfoun et al. (12) demonstraram que independentemente da origem, os grãos de café apresentaram elevada presença externa de fungos dos gêneros Aspergillus, Penicillium e Fusarium, o que também foi observado neste trabalho.

A diversidade de espécies de microrganismos associados a frutos e grãos de café pode estar relacionada ao tipo de processamento despolpado e natural, além do local de cultivo e das condições higiênico sanitária adotadas pelos produtores. Assim, o conhecimento das espécies é de grande importância para a definição de tecnologia para o processamento do café, através de técnicas que previnam a presença de microrganismos toxigênicos (25). Verifica-se neste trabalho, elevada incidência de Aspergillus, concordando com os resultados obtidos por Silva et al. (24), que também verificaram maior incidência do gênero Aspergillus, seguido pelos gêneros Penicillium, Fusarium e Cladosporium. O predomínio de Aspergillus spp. e de algumas espécies do gênero Penicillium em grãos de café ocorre devido ao caráter xerofílico desses microrganismos, que têm capacidade de se adaptar a condições de baixa umidade dos grãos (6). Espécies de Aspergillus como A. ochraceus, A. niger e A. flavus também são consideradas xerofílicas, desenvolvendo-se em condições de baixa atividade de água (22).

Não houve diferença estatística significativa entre os tratamentos, para as espécies Aspergillus ochraceus, A. niger e A. flavus em relação ao parâmetro I, em grãos não-desinfestados. Para o ISI, verifica-se que houve diferença estatística significativa apenas para a espécie A. niger, em grãos não-desinfestados, sendo que o tratamento natural de Encruzilhada apresentou o maior valor.

O grau de contaminação interna pode ser um indicador de nível de risco já existente nos grãos de café e qualquer variação ambiental favorável ao desenvolvimento dos fungos terá como consequência algum prejuízo cuja gravidade dependerá da espécie fúngica envolvida (15). A colonização interna pelos fungos pode ser explicada por danos causados por insetos, fungos fitopatogênicos, ácaros ou condições climáticas adversas, que tornam os grãos mais suscetíveis à incidência fúngica (4). Os resultados referentes aos parâmetros, I e ISI em grãos de café desinfestados, em função dos diferentes tratamentos, estão apresentados nas Tabelas 3 e 4, respectivamente.

Verifica-se que não houve diferença estatística significativa entre os tratamentos analisados para os gêneros Aspergillus, Penicillium e

Tabela 1. Valores médios de Incidência (\%) de fungos dos gêneros Aspergillus (Asp.), Penicillium (Pen.) e Fusarium (Fus.) e das espécies Aspergillus ochraceus, A. niger e A. flavus em grãos de café não-desinfestados, provenientes dos processamentos natural e despolpado dos municípios de Barra do Choça (BC) e Encruzilhada (EN), sudoeste da Bahia. Safra 2007/2008.

\begin{tabular}{|c|c|c|c|c|c|c|}
\hline \multirow{2}{*}{ Tratamentos } & \multicolumn{5}{|c|}{ Incidência (\%) } & \multirow[b]{2}{*}{ A.flavus } \\
\hline & Asp. & Pen. & Fus. & A.ochraceus & A.niger & \\
\hline Natural BC & 93,8 a & $31,4 \mathrm{a}$ & 5,6 a & 35,2 a & 42,2 a & $11,8 \mathrm{a}$ \\
\hline Despolpado BC & $74,0 \mathrm{~b}$ & $11,8 \mathrm{ab}$ & $0,2 \mathrm{a}$ & $3,6 \mathrm{a}$ & $68,4 \mathrm{a}$ & $13,4 \mathrm{a}$ \\
\hline Despolpado EN & $72,0 \mathrm{~b}$ & $8,0 \mathrm{~b}$ & $0,2 \mathrm{a}$ & $16,4 \mathrm{a}$ & $48,4 \mathrm{a}$ & $13,0 \mathrm{a}$ \\
\hline
\end{tabular}

* Médias seguidas pela mesma letra minúscula na coluna não diferem entre si pelo teste t de Bonferroni a $5 \%$ de probabilidade $(\mathrm{P}<0,05 \%)$.

Tabela 2. Valores médios de Índice de Severidade da Infestação (ISI) de fungos dos gêneros Aspergillus (Asp.), Penicillium (Pen.) e Fusarium (Fus.) e das espécies Aspergillus ochraceus, A. niger e A. flavus em grãos de café não-desinfestados, provenientes dos processamentos natural e despolpado dos municípios de Barra do Choça (BC) e Encruzilhada (EN), sudoeste da Bahia. Safra 2007/2008.

\begin{tabular}{|c|c|c|c|c|c|c|}
\hline \multirow{2}{*}{ Tratamentos } & \multicolumn{5}{|c|}{ Índice de Severidade da Infestação (ISI) } & \multirow[b]{2}{*}{ A.flavus } \\
\hline & Asp. & Pen. & Fus. & A.ochraceus & A.niger & \\
\hline Natural BC & $1,164 \mathrm{a}$ & $0,882 \mathrm{a}$ & 0,739 a & $0,911 \mathrm{a}$ & $0,930 \mathrm{~b}$ & 0,776 a \\
\hline Despolpado BC & $0,990 \mathrm{~b}$ & $0,777 \mathrm{~b}$ & 0,708 a & 0,731 a & $0,968 \mathrm{~b}$ & 0,772 a \\
\hline Despolpado EN & $0,978 \mathrm{~b}$ & $0,744 \mathrm{~b}$ & 0,708 a & $0,780 \mathrm{a}$ & $0,902 \mathrm{~b}$ & 0,768 a \\
\hline
\end{tabular}

* Médias seguidas pela mesma letra minúscula na coluna não diferem entre si pelo teste t de Bonferroni a $5 \%$ de probabilidade ( $\mathrm{P}<0,05 \%$ ). 
Tabela 3. Valores médios de Incidência (\%) de fungos dos gêneros Aspergillus (Asp.), Penicillium (Pen.) e Fusarium (Fus.) e das espécies Aspergillus ochraceus, A. niger e A. flavus em grãos de café desinfestados, provenientes dos processamentos natural e despolpado dos municípios de Barra do Choça (BC) e Encruzilhada (EN), sudoeste da Bahia. Safra 2007/2008.

\begin{tabular}{|c|c|c|c|c|c|c|}
\hline \multirow{2}{*}{ Tratamentos } & \multicolumn{5}{|c|}{ Incidência (\%) } & \multirow[b]{2}{*}{ A. flavus } \\
\hline & Asp. & Pen. & Fus. & A.ochraceus & A.niger & \\
\hline Natural BC & $64,2 \mathrm{a}$ & $2,6 \mathrm{a}$ & $2,4 \mathrm{a}$ & $1,4 \mathrm{a}$ & $46,2 \mathrm{a}$ & $1,2 \mathrm{a}$ \\
\hline Natural EN & 64,8 a & $1,6 \mathrm{a}$ & $0,6 \mathrm{a}$ & $1,0 \mathrm{a}$ & 48,4 a & $2,4 \mathrm{a}$ \\
\hline Despolpado BC & 69,6 a & $2,6 \mathrm{a}$ & $0,0 \mathrm{a}$ & $1,2 \mathrm{a}$ & 49,8 a & $1,8 \mathrm{a}$ \\
\hline Despolpado EN & $67,0 \mathrm{a}$ & $1,8 \mathrm{a}$ & $0,0 \mathrm{a}$ & 0,8 a & $40,2 \mathrm{a}$ & $1,2 \mathrm{a}$ \\
\hline $\mathrm{CV}(\%)$ & 10,82 & 12,15 & 13,11 & 10,02 & 14,23 & 13,17 \\
\hline
\end{tabular}

* Médias seguidas pela mesma letra minúscula na coluna não diferem entre si pelo teste t de Bonferroni a $5 \%$ de probabilidade (P<0,05\%).

Tabela 4. Valores médios de Índice de Severidade da Infestação (ISI) de fungos dos gêneros Aspergillus (Asp.), Penicillium (Pen.) e Fusarium (Fus.) e das espécies Aspergillus ochraceus, A. niger e A. flavus em grãos de café desinfestados, provenientes dos processamentos natural e despolpado dos municípios de Barra do Choça (BC) e Encruzilhada (EN), sudoeste da Bahia. Safra 2007/2008.

\begin{tabular}{|c|c|c|c|c|c|c|}
\hline \multirow{2}{*}{ Tratamentos } & \multicolumn{5}{|c|}{ Índice de Severidade da Infestação (ISI) } & \multirow[b]{2}{*}{ A.flavus } \\
\hline & Asp. & Pen. & Fus. & A.ochraceus & A.niger & \\
\hline Natural BC & $0,995 \mathrm{a}$ & $0,721 \mathrm{a}$ & $0,720 \mathrm{a}$ & $0,714 \mathrm{a}$ & $0,927 \mathrm{a}$ & $0,714 \mathrm{a}$ \\
\hline Natural EN & 0,995 a & $0,714 \mathrm{a}$ & 0,709 a & $0,714 \mathrm{a}$ & 0,935 a & 0,721 a \\
\hline Despolpado BC & $0,980 \mathrm{a}$ & 0,723 a & $0,000 \mathrm{a}$ & 0,715 a & 0,920 a & 0,728 a \\
\hline Despolpado EN & 0,975 a & 0,718 a & $0,000 \mathrm{a}$ & $0,712 \mathrm{a}$ & $0,900 \mathrm{a}$ & $0,714 \mathrm{a}$ \\
\hline $\mathrm{CV}(\%)$ & 5,64 & 0,91 & 1,05 & 0,66 & 6,21 & 2,26 \\
\hline
\end{tabular}

* Médias seguidas pela mesma letra minúscula na coluna não diferem entre si pelo teste $\mathrm{t}$ de Bonferroni a $5 \%$ de probabilidade $(\mathrm{P}<0,05 \%)$.

Fusarium em relação aos parâmetros I e ISI, em grãos desinfestados superficialmente. Isso demonstra que, independentemente do processamento pós-colheita, os grãos de café apresentaram uma contaminação fúngica semelhante de cada gênero e provavelmente oriunda da lavoura. Após a desinfestação dos grãos não houve a ocorrência de fungos do gênero Fusarium em grãos de café despolpados dos municípios de Barra do Choça e Encruzilhada.

Verifica-se também que não houve diferença estatística significativa entre os tratamentos, para as espécies Aspergillus ochraceus, A. niger e A. flavus em relação ao parâmetros I e ISI, em grãos desinfestados. Entretanto, grãos com níveis acima de $5 \%$ de infecção podem representar risco ao armazenamento do café (15). Considerando este limite de tolerância de infecção interna dos grãos, observa-se que as espécies A. ochraceus e A.flavus apresentaram níveis, em média geral, inferiores a este valor. Entretanto, a espécie $A$. niger apresentou níveis de infecção interna superiores a $5 \%$, com incidência variando entre 40,2 e 49,8\%. Pereira (21) caracterizou as diferentes comunidades de fungos presentes em frutos e grãos de café e detectou, dentro do gênero Aspergillus, as espécies A. ochraceus e A. niger em $66 \%$ e $60 \%$ das amostras, respectivamente. Em outro estudo, amostras de café secas em terreiro de cimento, via processamento natural, apresentaram sete diferentes espécies de Aspergillus, dentre elas, A. niger ocorreu em maior percentual (26).

A presença de espécies de Aspergillus com potencial toxigênico também tem sido detectada em outras regiões do País. Martins et al. (18) verificaram que $91,7 \%$ das amostras de grãos de café do Brasil estavam contaminadas com fungos, incluindo $A$. niger (83,3\%), A. ochraceus $(53,3 \%)$ e A.flavus $(25,0 \%)$. Pasin et al. (20) verificaram que a ocorrência de A. niger e A. ochraceus não diferiu, em grãos processados por via seca, entre as cultivares de café e que em nenhuma amostra foi detectada a ocorrência de ocratoxina A. Magnani et al. (17) detectaram a presença de espécies potencialmente toxigênicas em $82 \%$ das regiões estudadas no norte do Estado do Paraná, entre as quais Aspergillus niger foi a espécie mais frequentemente detectada, seguida por A. ochraceus e $A$. carbonarius.

A maior incidência de fungos do gênero Aspergillus, Seção Nigri, observada neste trabalho, corrobora aquela observada por Batista et al. (6), os quais encontraram contaminação com Aspergillus em 95,55\% das amostras sem desinfestação e 46,66\% das amostras após a desinfestação. Antes da desinfestação, a maior incidência foi de fungos da Seção Nigri, presente em $88,37 \%$ das amostras, seguida pela Seção Circumdati, presente em $65,12 \%$ e pela Seção Flavi, presente em $44,19 \%$ das amostras. Após a desinfestação, também houve predomínio das espécies pertencentes à Seção Nigri, presente em $25 \%$ das amostras, seguida pela Seção Circumdati, presente em 16,27\% e Seção Flavi, presente em 6,98\% das amostras. Pasin et al. (20), em estudo semelhante, constataram que a incidência de fungos no interior dos grãos foi significativamente menor à encontrada externamente, em cafés de diferentes cultivares processados por via seca. Segundo SuárezQuiroz et al. (27), os isolados de Aspergillus ochraceus representaram $6,6 \%$ do processo úmido, $8,3 \%$ do processo mecânico e 3,3\% do processo de seca natural e os isolados de Aspergillus niger representaram $15 \%$ do processo úmido, $13 \%$ do processo mecânico e $25 \%$ do processo de seca natural.

Desta forma, pôde-se verificar que o processamento natural de café, com secagem em terreiro de terra, proporcionou maior infestação fúngica que o processamento despolpado, com secagem em terreiro de cimento, independentemente do local de origem, havendo a ocorrência de fungos dos gêneros Aspergillus, Penicillium e Fusarium em grãos de café beneficiados, com elevada incidência 
do gênero Aspergillus. Foram identificadas também, as espécies Aspergillus ochraceus, A. niger, A. flavus, A. foetidus, A. tubingensis, A. auricomus, A. sojae e A. oryzae associadas a grãos de café beneficiados oriundos da região sudoeste da Bahia.

\section{REFERÊNCIAS BIBLIOGRÁFICAS}

1. Alves, E.; Castro, H.A.de. Fungos associados ao café (Coffea arabica L.) nas fases de pré e pós-colheita em lavouras da região de Lavras. Summa Phytopathologica, Botucatu, v.24, n.1, p.4-7, 1998.

2. Barnett, H.L.; Hunter, B.B. Illustrated genera of imperfect fungi. 4. ed. Minneapolis: Burgess Publishing, 1998. 218 p.

3. Batista, L.R.; Chalfoun, S.M.; Silva, C.F.; Cirillo, M.; Varga, E.A.; Schwan, R.F. Ochratoxin A in coffee beans (Coffea arabica L.) processed by dry and wet methods. Food Control, Guildford, v.20, n.9 p.784-790, 2009.

4. Batista, L.R.; Chalfoun, S.M. Incidência de Ocratoxina A em diferentes frações do café (Coffea arabica L.): bóia, mistura e varrição após secagem em terreiros de terra, asfalto e cimento. Ciência e Agrotecnologia, Lavras, v.31, n.3, p.804-813, 2007.

5. Batista, L.R.; Chalfoun, S.M.; Prado, G.; Schwan, R.F.; Wheals, A.E. Toxigenic fungi associated with processed (green) coffee beans (Coffea arabica L.). International Journal of Food Microbiology, Amsterdam, v.85, n.3, p.293-300, 2003.

6. Batista, L.R.; Chalfoun, S.M.; Prado, G. Identificação de espécies toxigênicas de Aspergillus associados aos grãos de café armazenados. Revista Brasileira de Armazenamento, Viçosa, n.3, p.11-16, 2001. Especial Café.

7. Bucheli, P.; Taniwaki, M.H. Research on the origin, and the impact of post-harvest handling and manufacturing on the presence of ochratoxin A in coffee - Review. Food Additives and Contaminants, London, v.19, n.7, p.655-665, 2002.

8. Campos, R. da S.; Freitas-Silva, O.; Cunha, F.Q. da; Souza, M. de L.M. de; Freitas, S.C. de. Fungos micotoxigênicos e ocratoxina A em cafés com permanência prolongada na planta e no solo, colhidos nas regiões do cerrado mineiro e baiano. Coffee Science, Lavras, v.4, n.2, p.136-148, 2009.

9. Chalfoun, S.M.; Parizzi, F.C. Fungos toxigênicos e micotoxinas em café. In: Borém, F.M. Pós-colheita do café. Lavras: Ed. UFLA, 2008. cap. 14, p.512-543.

10. Chalfoun, S.M.; Batista, L.R. Incidência de Ocratoxina A em diferentes frações de grãos de café (Coffea arabica L.). Coffee Science, Lavras, v.1, n.1, p.28-35, 2006.

11. Chalfoun, S.M.; Batista, L.R. Fungos associados a frutos e grãos de café Aspergillus \& Penicillium. Brasília, DF: Embrapa, 2003. 69 p.

12. Chalfoun, S.M.; Chagas, S.J. de R.; Pereira, M.C. Determinação da microbiota associada externa e internamente a grãos beneficiados de café. Summa Phytopathologica, Botucatu, v.25, n.4, p.369-372, 1999.

13. Dutra Neto, C. Desenvolvimento regional e agronegócio. Vitória da Conquista: Ed. do Autor, 2009. 188 p.

14. Ferreira, D.F. Sisvar 4.3. Lavras: DEX-UFLA, 2000. Disponível em: <http://www.dex.ufla.br >.
15. Freitas, R.F.de. Fungos associados a grãos de café (Coffea arabica $L$.) beneficiado de diversos municípios da região Sul de Minas Gerais. 2000. 72 p. Dissertação (Mestrado em Ciência dos Alimentos) - Universidade Federal de Lavras, Lavras.

16. Klich, M.A. Identification of Common Aspergillus species. Utrecht: Centraalbureau voor Schimmelculture, 2002. 116 p.

17. Magnani, M.; Fernandes, T.; Prete, C.E.C; Homechim, M.; Ono, E.Y.S; Vilas-Boas, L.A.; Sartori, D; Furlaneto, M.C.; Fungaro, M.H.P. Molecular identification of Aspergillus spp. isolated from coffee beans. Scientia Agricola, Piracicaba, v.62, n.1, p.45-49, 2005.

18. Martins, M.L.; Martins, H.M.; Gimeno, A. Incidence of microflora and of ochratoxin A in green coffee beans (Coffea arabica). Food Additives and Contaminants, London, v.20, n.12, p.1127-1131, 2003.

19. Moraes, M.L.P.; Luchese, R.H. Ochratoxin A on green coffee: influence of harvest and drying processing procedures. Journal of Agricultural and Food Chemistry, Easton, v.51, n.19, p.5824-5828, 2003.

20. Pasin, L.A.A.P.; Almeida, J.R. de; Abreu, M.S. de. Fungos associados a grãos de cinco cultivares de café (Coffea arabica L.). Acta Botanica Brasilica, Porto Alegre, v.23, n.4, p.1129-1132, 2009.

21. Pereira, R.T.G. Diversidade e freqüência de fungos associados a frutos e grãos de café. 2006. 151 f. Tese (Doutorado em Agronomia - Fitopatologia) - Universidade Federal de Lavras, Lavras.

22. Pitt, J.I.; Hocking, A.D. Fungi and food spoilage. London: Blackie Academic \& Professional, 1997. 175 p.

23. Prabhu, A.S.; Bedendo, I.P. Glume blight of rice in Brazil: etiology, varietal, reaction and loss estimates. Tropical Pest Management, London, v.34, n.1, p.85-88, 1988.

24. Silva, C.F.; Batista, R.F.; Schwan, R.F. Incidence and distribution of filamentous fungi during fermentation, drying and storage of coffee (Coffea arabica L.) beans. Brazilian Journal of Microbiology, São Paulo, v.39, n.1, p.521-526, 2008.

25. Silva, C.F.; Schwan, R.F.; Dias, E.S.; Wheals, A.E. Microbiota presente em frutos e grãos de café despolpado e natural - uma revisão. Boletim da Sociedade Brasileira de Ciência e Tecnologia de Alimentos, Campinas, v.37, supl., p.22-28, 2003a.

26. Silva, C.F.; Batista, L.R.; Schwan, R.F. Incidência de Aspergillus produtores de micotoxinas em frutos e grãos de café (Coffea arabica L.). Revista Brasileira de Armazenamento, Viçosa, n.7, p.30-36, 2003b. Especial Café.

27. Suárez-Quiroz, M.L.; González-Rios, O.; Barel, M.; Guyot, B.; Schorr-Galindo, S.; Guiraud, J.P. Study of ochratoxin A- producing strains in coffee processing. International Journal of Food and Technology, Oxfford, v.39, n.5, p.501-507, 2004

28. Taniwaki, M.H.; Pitt, J.I.; Teixeira, A.A.; Iamanaka, B.T. The source of ochratoxin A in Brazilian coffee and its formation in relation to processing methods. International Journal of Food Microbiology, Amsterdam, v.82, n.2, p.173-179, 2003.

29. Tempe, J. de. The blotter method for seed health testing. Proceeding of the International Seed Testing Association, Vollebekk, v.28, n.1, p.133-151, 1963.

30. Urbano, G.R.; Taniwaki, M.H.; Leitão, M.F. de F.; Vicentini, M.C. Occurrence of ochratoxin A - Producing fungi in raw Brazilian coffee. Journal of Food Protection, Des Moines, v.64, n.8, p.1226-1230, 2001 